\title{
Comparison of the outcomes of nasal bone reduction using serial imaging
}

Cho Long Lee',

Ho Jik Yang ${ }^{2}$, Young Joong Hwang ${ }^{1}$

${ }^{1}$ Department of Plastic and

Reconstructive Surgery, Eulji University

Hospital, Daejeon; ${ }^{2}$ Department of

Plastic and Reconstructive Surgery,

Chungnam National University

Hospital, Daejeon, Korea

\begin{abstract}
Background: Nasal bone fractures are frequently encountered in clinical practice. Although fracture reduction is simple and correction requires a short operative time, low patient satisfaction and relatively high complication rates remain issues for many surgeons. These challenges may result from inaccuracies in fracture recognition and assessment or inappropriate surgical planning. Findings from immediate postoperative computed tomography (CT) scans and those performed at 4 to 6 weeks postoperatively were compared to evaluate the accuracy and outcomes of nasal fracture reduction.

Methods: This retrospective study included patients diagnosed with nasal bone fractures at our department who underwent closed reduction surgery. Patients who did not undergo additional CT scans were excluded from the study. Clinical examinations, patient records, and radiographic images were evaluated in 20 patients with nasal bone fractures.

Results: CT findings from immediately after surgery and a 1month follow-up were compared in 20 patients. Satisfactory nasal projection and aesthetically acceptable results were observed in patients with accurate correction or mild overcorrection, while undercorrection was associated with unfavorable results.

Conclusion: Closed reduction surgery for correcting nasal bone fractures usually provides acceptable outcomes with relatively few complications. If available, immediate postoperative CT scans are recommended to guide surgeons in the choice of whether to perform secondary adjustments if the initial results are unsatisfactory. Based on photogrammetric data, nasal bone reduction with accurate correction or mild overcorrection achieved acceptable and stable outcomes at 1 month postoperatively. Therefore, when upward dislocation is observed on postoperative CT, one can simply observe without a subsequent intervention.
\end{abstract}

Abbreviations: CT, computed tomography; SD, step deformity.

Keywords: Closed fracture reduction / Facial bones / Nasal bone

\section{INTRODUCTION}

Fractures of the nasal bone account for approximately $40 \%$ of all facial fractures and are the third most common type of skeletal fracture [1]. Nasal bone fractures should be diagnosed

Correspondence: Young Joong Hwang

Department of Plastic and Reconstructive Surgery, Eulji University Hospital,

95 Dunsanseo-ro, Seo-gu, Daejeon 35233, Korea

E-mail: yjhmedi@nate.com

Received July 20, 2021 / Revised August 6, 2021 / Accepted August 20, 2021 promptly and corrected using appropriate reduction techniques for optimal recovery. These fractures are sometimes dismissed as minor injuries; however, if diagnosis and treatment are overlooked, they can lead to nasal deformities [2]. Failure to perform timely fracture treatment can cause severe functional and cosmetic impairment, affecting the patient's mental health and social life. However, because reduction techniques for treating nasal bone fractures are usually non-invasive, ensuring accurate reduction is challenging. Postoperative imaging is performed to 
observe the degree of fracture reduction and assess whether the reduction is complete, but a thorough postoperative evaluation is often neglected because reduction is generally considered to be a simple procedure. Plain radiography alone has limited utility for detecting fractures and assessing their severity. Therefore, studies have suggested that postoperative computed tomography (CT) scans can be used to evaluate patients' postoperative condition, including the reduction status of the fracture and the location of the fracture fragment $[3,4]$. CT scans also help patients visualize and understand the fractures [5-8]. In addition, clinicians can observe whether the external shape of the nose improves or deteriorates after complete or incomplete reduction.

This study investigated changes in the degree of fracture reduction and the position of fracture fragments on CT images taken immediately after surgery and during short-term followup, with the goal of evaluating the outcomes of bone remodeling.

\section{METHODS}

\section{Patient selection}

This retrospective study was approved by the Institutional Review Board of Eulji University Hospital. Demographic and clinical information and imaging findings (radiography and CT) were obtained for patients who underwent closed reduction surgery at our institution for pure nasal bone fractures from August 2020 to February 2021. This study was limited to patients who underwent CT immediately after surgery and at a follow-up consultation 4 to 6 weeks later. Patients unwilling to undergo $\mathrm{CT}$ evaluations due to concerns regarding radiation exposure were excluded. Twenty nasal fracture patients who underwent manual revision surgery and follow-up CT scans 4 to 6 weeks later were included in this study. The age distribution was 16 to 59 years, and the male-to-female ratio was 13:7.

\section{Surgical technique}

In each patient, the nasal fracture was reduced using closed reduction. Surgery was performed within 2 weeks of the injury under general anesthesia. Depressed bone fractures were manipulated outwards, while convex bone fractures were manipulated inwards. After restoration of the fracture, cotton gauze was inserted in the upper part of the nasal cavity to maintain the reduced state of the fracture fragment. An external nasal Thermo-Splint was applied.

Intranasal fillings, such as Merocel (Medtronic Xomed, Jacksonville, FL, USA) or Vaseline roll gauze, were not provided. CT scans were performed within 24 hours after surgery. The nasal cotton gauze was removed 72 hours postoperatively, and the nasal splint was worn for 4 weeks. None of the 20 patients underwent a second operation or revision by blind manual reduction in the ward. CT images were obtained 4 to 6 weeks after surgery.

\section{Assessment methods}

CT scans were performed using was a 128-channel CT device (Somatom Definition AS+; Siemens Healthcare, Erlangen, Germany). Axial CT images were performed with $2-\mathrm{mm}$ slices. The images encompassed the area from the tip of the nose to the entire area around the nasal bone. The same or most similar image sections were compared and observed for each patient. Photogrammetric analysis was performed by the same rater using the ImageJ 1.50i image analysis software (National Institutes of Health, Bethesda, MD, USA).

The evaluation was based on three factors: bony contact, deviation angle, and arch shape. The evaluation results were classified into the following four grades: (1) excellent: step deformity (SD) 0 (bony contact $75 \%-100 \%$ ), deviation angle $0^{\circ}-20^{\circ}$, smooth arch shape; (2) good: SD+ (bony contact 25\%-75\%), deviation angle $0^{\circ}-20^{\circ}$, almost normal arch shape; (3) fair: SD++ (bony contact under $25 \%$ ), deviation angle over $20^{\circ}$, irregular arch shape; or (4) poor: SD-, SD-- (undercorrected reduction), any deviation, any arch shape.

The reduction surgery outcome of each patient was classified as excellent, good, fair, or poor based on the immediate postoperative CT findings (Table 1). Images obtained 4 to 6 weeks after surgery were classified similarly. The most important parameter was bony contact. The degree of deviation of the fracture fragments was classified based on the SD. The effect of the $\mathrm{SD}$ on the outcomes of surgery was also analyzed. Three plastic surgeons evaluated the grade of the fracture, and the outcome of bone remodeling was graded based on a majority vote to ensure an objective comparison.

\section{RESULTS}

The immediate postoperative CT scan findings revealed excellent results in three of the 20 patients (15\%), good results in nine patients (45\%), fair results in six patients (30\%), and poor results in two patients (10\%). In contrast, the findings of the CT scans performed at 4 to 6 weeks postoperatively revealed excellent results in 10 patients (50\%), good results in seven patients (35\%), fair result in one patient (5\%), and poor results in two patients (10\%). The progression of patients' outcomes between these two-time points was as follows: excellent to excellent in three patients (15\%), good to excellent in five patients (25\%), 
Table 1. Parameters for reduction assessment

\begin{tabular}{|c|c|c|}
\hline \multirow[t]{6}{*}{ Step deformity (SD) } & & Normal \\
\hline & $\mathrm{SD}++$ & Non-contact outward displacement from the maxillary frontal process (bony contact under 25\%) \\
\hline & $\mathrm{SD}+$ & Half-contact outward displacement from the maxillary frontal process (bony contact $25 \%-75 \%$ ) \\
\hline & SDO & Closed contact to maxillary frontal process (bony contact $75 \%-100 \%$ ) \\
\hline & SD- & Half-contact inward displacement from maxillary frontal process (undercorrected reduction) \\
\hline & SD-- & Non-contact inward displacement from maxillary frontal process (undercorrected reduction) \\
\hline \multicolumn{3}{|l|}{ Deviation angle } \\
\hline & Good & Angulated fragments show deviation of less than $20^{\circ}$, smooth connection \\
\hline & Poor & Angulated fragments show deviation of more than $20^{\circ}$, irregular connection \\
\hline \multirow[t]{2}{*}{ Arch shape } & Smooth & \\
\hline & Irregular & \\
\hline \multirow[t]{4}{*}{ Gross assessment } & Excellent & SD0 (bony contact $75 \%-100 \%$ ), deviation angle $0^{\circ}-20^{\circ}$, smooth arch shape \\
\hline & Good & $\mathrm{SD}+$ (bony contact $25 \%-75 \%$ ), deviation angle $0^{\circ}-20^{\circ}$, almost normal arch shape \\
\hline & Fair & $\mathrm{SD}++$ (bony contact under $25 \%$ ), deviation angle over $20^{\circ}$, irregular arch shape \\
\hline & Poor & SD-, SD-- (undercorrected reduction), any deviation, any arch shape \\
\hline
\end{tabular}

Table 2. Results of case 1

\begin{tabular}{lll}
\hline Result & Postoperative findings & Follow-up findings (4 weeks) \\
\hline Bony contact & Left $(++)$, right $(++)$ & Left (0), right (0) \\
Angle of deviation & Poor & Good \\
Arch shape & Irregular & Smooth \\
Gross assessment & Fair & Excellent \\
\hline
\end{tabular}

$0,++$ : the degree of step deformity.

fair to excellent in two patients (10\%), fair to good in three patients (15\%), fair to fair in one patient (5\%), good to good in four patients (20\%), excellent to fair or poor in 0 patients, and good to fair or poor in 0 patients (Figs. 1-3), and poor to poor in two patients (10\%). Of note, neither of the two patients with poor results on the immediate postoperative CT scan showed improvement 4 to 6 weeks after surgery.

\section{Case 1}

A 19-year-old man presented to the hospital with epistaxis and facial swelling due to a motorcycle accident (Table 2). CT revealed a nasal bone fracture. After closed reduction surgery, immediate postoperative CT images showed mild overcorrection of the bilateral walls of the nasal bone. A follow-up CT examination showed excellent alignment of the fractured seg-
Table 3. Results of case 2

\begin{tabular}{lll}
\hline Result & Postoperative findings & Follow-up findings (5 weeks) \\
\hline Bony contact & Left $(++)$, right $(0)$ & Left (0), right (0) \\
Angle of deviation & Good & Good \\
Arch shape & Irregular & Smooth \\
Gross assessment & Fair & Excellent \\
\hline
\end{tabular}

$0,++$ : the degree of step deformity.

Table 4. Results of case 3

\begin{tabular}{lll}
\hline Result & Postoperative findings & Follow-up findings (4 weeks) \\
\hline Bony contact & Left (0), right (0) & Left (-), right (0) \\
Angle of deviation & Good & Good \\
Arch shape & Smooth & Smooth \\
Gross assessment & Excellent & Good \\
\hline
\end{tabular}

$0,-:$ the degree of step deformity.

ments. Immediate postoperative $\mathrm{CT}$ images revealed fair results of reduction, which progressed to excellent on follow-up CT (Fig. 1).

\section{Case 2}

A 26-year-old man presented to our outpatient department with nasal swelling and bruising after a slipping accident (Table 3). 

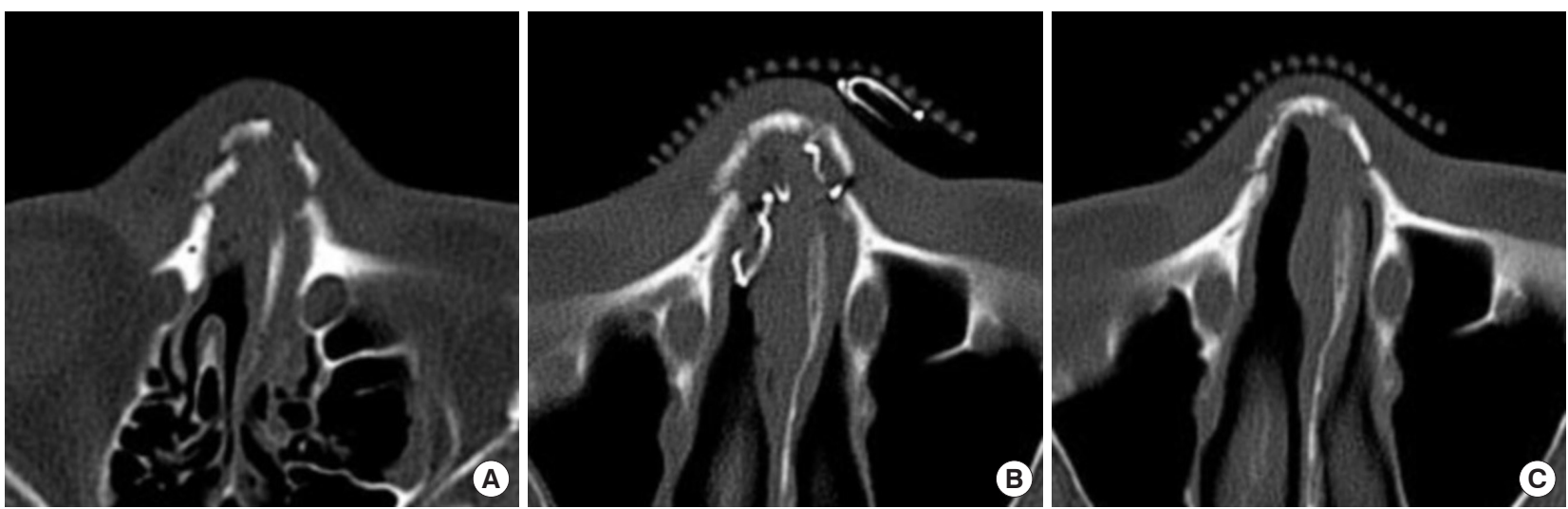

Fig. 1. Computed tomographic images of a 19-year-old man who presented to our clinic after a motorcycle accident. (A) Preoperative findings showing a nasal bone fracture. (B) Postoperative findings showing excellent alignment of the fractured segment. (C) Four-week follow-up findings showing excellent results.
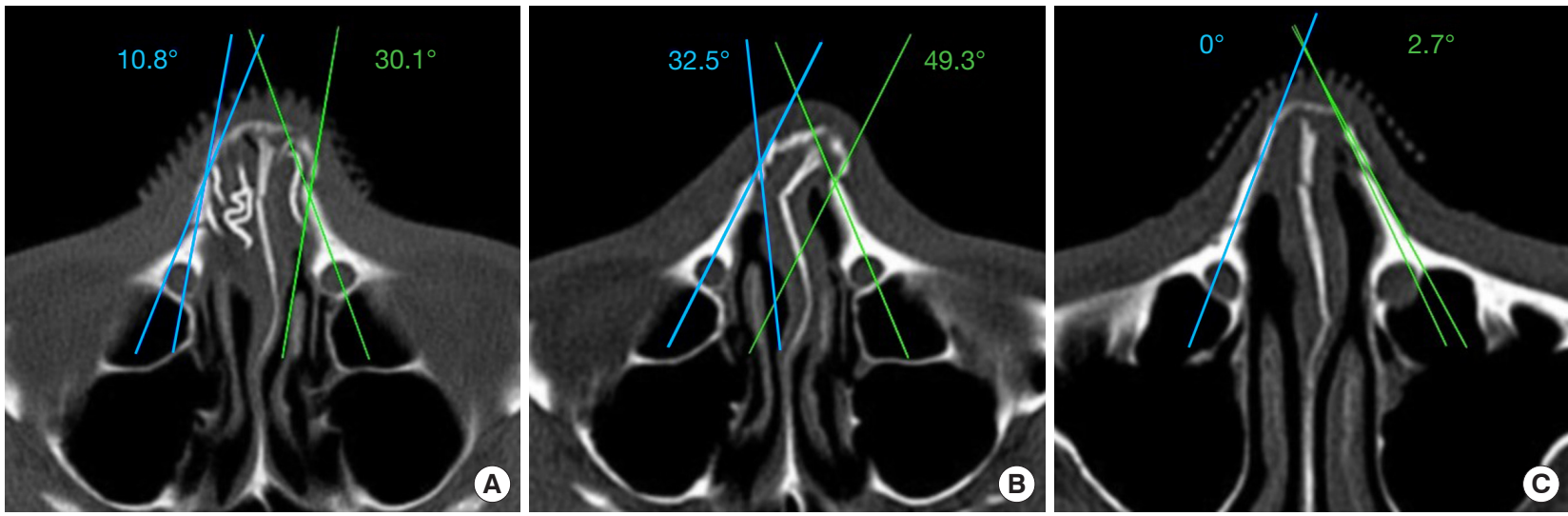

Fig. 2. Computed tomographic images of a 26-year-old man who presented with nasal swelling and bruising after a slipping accident. (A) Preoperative findings showing a nasal bone fracture. (B) Postoperative findings showing mild overcorrection of the fractured segments. (C) Fiveweek follow-up findings showing excellent alignment of the segments. The blue and green angles are measurements showing the changes in the angle of deviation.
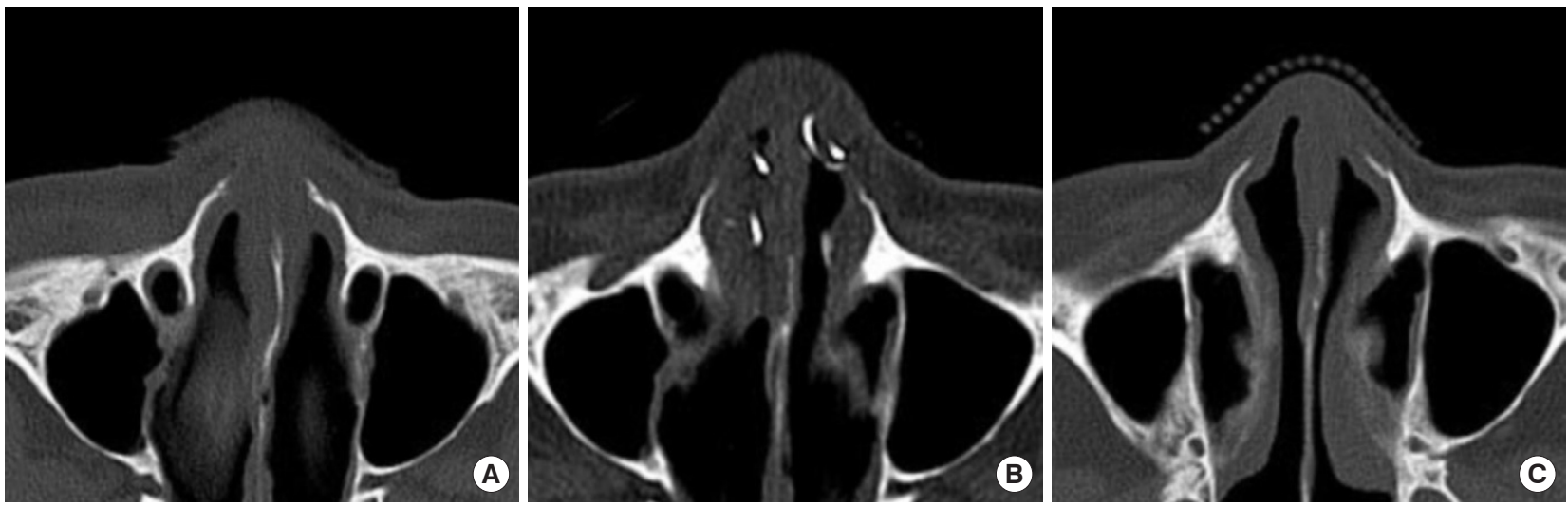

Fig. 3. Computed tomographic images of a 45-year-old woman with a nasal bone fracture. (A) Preoperative findings showing fracture of the nasal bone. (B) Postoperative findings showing undercorrection of the left lateral wall. (C) Five-week follow-up findings showing no change in the fractured segment.

He was diagnosed with a nasal bone fracture and underwent closed reduction surgery. As shown in Fig. 2, immediate post- operative CT images demonstrated mild overcorrection of the fractured segments. After 5 weeks, follow-up CT images showed 
excellent alignment of the segments. The outcome of this patient changed from fair to excellent during follow-up (Fig. 2).

\section{Case 3}

A 45-year-old woman was diagnosed with a nasal bone fracture and underwent closed reduction surgery at our department (Table 4). Immediate postoperative CT images showed that the left lateral wall depression was undercorrected with poor alignment. Follow-up CT scans performed postoperatively at the fourth week revealed slight depression of the fractured segment. Therefore, the outcomes of this patient remained poor during follow-up (Fig. 3).

\section{DISCUSSION}

Fractures of the nasal bone are the most common type of facial fracture; therefore, they are frequently encountered in the field of plastic surgery $[9,10]$. Reduction to correct nasal fractures is easy, simple, and non-invasive, and postoperative imaging is frequently overlooked for these reasons. In some patients, the appearance of the nose improves with a decrease in edema over time. Conversely, no improvement is observed in other patients, and the deformation persists. A deformity of the nose that develops immediately after surgery may be due to an incomplete perioperative reduction of the fracture or a positional abnormality due to postoperative movement of the reduced fracture fragment-as such, it may improve or persist over time. Because of the uncertain long-term results, the plastic surgeon may be tempted to conduct revision by manual adjustment in the ward or at an outpatient facility after the surgery.

In the present study, postoperative changes in the location of the fracture fragments were evaluated using CT scans to assess the progression of bone remodeling using follow-up CT scans, which have been established as a useful modality for this purpose $[5,6]$, and to determine the clinical significance of changes during this period. It could be helpful to use serial CT scans to assess the outcomes of nasal bone fracture correction in strictly selected patients, but the radiation dose of CT is higher than that of simple X-rays; therefore, the procedure should be done only for research aims, with appropriate consideration of risk and benefits, after receiving approval from an ethical committee. Although the number of study participants was limited, there were no cases in this study in which the patients' outcome regressed from excellent to good, fair, or poor; or from good to fair or poor. Therefore, it can be assumed that the postoperative deformities were a consequence of insufficient reduction. The outcomes of five patients progressed from fair to good or excellent, and those of five patients progressed from good to excel- lent. The outcomes of seven patients, which were classified as good or fair (including SD+ and SD++), progressed to excellent. Notably, there were no improvements in the outcomes of patients with undercorrection.

These results suggest that postoperative deformities develop due to insufficient correction during surgery and not due to the depression of fracture fragments occurring early after surgery. Accurate corrections of the fracture fragments were well maintained and showed excellent results. Minimally overcorrected fragments progressed to excellent; however, undercorrected fragments remained undercorrected. Therefore, accurate reduction is the best operation, but in overcorrected cases, there is no need for surgeons to correct the deficiencies. However, in cases of undercorrection, clinicians should consider a second operation or revision using manual adjustment in the ward. However, further studies with larger study populations are required to provide empirical support for this proposal.

Schultz and deVillers [11] pointed out that a fractured fragment reduced to an appropriate position can maintain the pyramidal shape of the external nose through a self-supporting force. Additionally, some cases of dislodgement of fracture fragments immediately after surgery improve after 1 month because the nasal bone is membranous and the connections between the remaining periosteum and connective tissue are well maintained [12,13].

The remodeling process of a membranous bone is longer than that of a long bone. After surgery, pressure applied to the fracture site causes gradual remodeling, leading to a better shape of bone union. Therefore, it can be inferred that outward malposition in a nasal bone reduction is more beneficial for post-surgical outcomes than downward malposition. In particular, the use of an external fixation device may be important for preventing postoperative swelling, providing continuous symmetrical pressure, and eliminating external forces on the outside of the nose.

It is equally important to keep the fracture fragment stable after surgery. If an upwardly displaced fracture fragment is subjected to blind manual reduction to induce downward displacement, favorable outcome progression is unlikely. Therefore, if upward dislocation is observed on CT after surgery, it is recommended to simply observe the case without subsequent intervention.

Thus, it is essential to perform surgery carefully and precisely, to evaluate the results postoperatively, to explain the injury and treatment outcomes to the patient, and to conduct adequate follow-up assessments because nasal bone fractures can change over time. To achieve optimal progression, care must be taken to avoid positioning the fracture fragment downwards during 
or after surgery.

Nonetheless, the current study has some limitations. The small number of subjects may make it difficult to generalize our findings. Simple X-ray examinations can be performed instead of CT scans due to radiation concerns, although they are more difficult to analyze.

\section{NOTES}

\section{Conflict of interest}

No potential conflict of interest relevant to this article was reported.

\section{Ethical approval}

The study was approved by the Institutional Review Board of Eulji University Hospital (IRB No. 2021-07-004) and performed in accordance with the principles of the Declaration of Helsinki. Written informed consent was obtained.

\section{Patient consent}

The patients provided written informed consent for the publication and the use of their images.

\section{ORICD}

Cho Long Lee https://orcid.org/0000-0002-6441-0514 Ho Jik Yang https://orcid.org/0000-0003-2354-3983

Young Joong Hwang https://orcid.org/0000-0001-7349-7132

\section{Author contribution}

Conceptualization: HJY, YJH. Data curation: CLL. Visualization: CLL. Writing - original draft: CLL. Writing - review \& editing: HJY. Supervision: YJH.

\section{REFERENCES}

1. Kucik CJ, Clenney T, Phelan J. Management of acute nasal fractures. Am Fam Physician 2004;70:1315-20.
2. Han DG, Kim TS. The effect of half day nasal packing in results of closed reduction of nasal bone fracture. Arch Craniofac Surg 2012;13:119-24.

3. Choi MH, Cheon JS, Son KM, Choi WY. Long-term postoperative satisfaction and complications in nasal bone fracture patients according to fracture type, site, and severity. Arch Craniofac Surg 2020;21:7-14.

4. Park YJ, Ryu WS, Kwon GH, Lee KS. The clinical usefulness of closed reduction of nasal bone using only a periosteal elevator with a rubber band. Arch Craniofac Surg 2019;20:284-8.

5. Manson PN, Markowitz B, Mirvis S, Dunham M, Yaremchuk M. Toward CT-based facial fracture treatment. Plast Reconstr Surg 1990;85:202-12.

6. Hwang K, You SH, Kim SG, Lee SI. Analysis of nasal bone fractures; a six-year study of 503 patients. J Craniofac Surg 2006; 17:261-4.

7. Pollock RA. Nasal trauma: pathomechanics and surgical management of acute injuries. Clin Plast Surg 1992;19:133-47.

8. Yabe T, Ozawa T, Sakamoto M, Ishii M. Pre- and postoperative $\mathrm{x}$-ray and computed tomography evaluation in acute nasal fracture. Ann Plast Surg 2004;53:547-53.

9. Muraoka M, Nakai Y. Twenty years of statistics and observation of facial bone fracture. Acta Otolaryngol Suppl 1998;538: 261-5.

10. Han DG. Considerations for nasal bone fractures: preoperative, perioperative, and postoperative. Arch Craniofac Surg 2020; 21:3-6.

11. Schultz RC, deVillers YT. Nasal fractures. J Trauma 1975;15: 319-27.

12. Sekido M, Sasaki S, Chiba M, Sugihara T, Ohura T. A study of nasomaxillary bone defect repair: the role of nasal mucosa and periosteum in such repair. J Jpn Soc Plast Reconstr Surg 1995; 15:148-56

13. Dahlin C, Linde A, Gottlow J, Nyman S. Healing of bone defects by guided tissue regeneration. Plast Reconstr Surg 1988;81:6726. 